\title{
Numerical experiments on plasma focus pinch current limitation
}

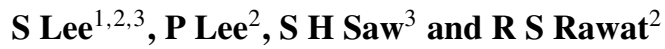 \\ ${ }^{1}$ Institute for Plasma Focus Studies, 32 Oakpark Drive, Chadstone, VIC 3148, Australia \\ 2 Nanyang Technology University, National Institute of Education, Singapore 637616, \\ Singapore \\ ${ }^{3}$ INTI International University College, 71800 Nilai, Malaysia \\ E-mail: leesing@optusnet.com.au
}

Received 18 February 2008

Published 11 April 2008

Online at stacks.iop.org/PPCF/50/065012

\begin{abstract}
Contrary to the general expectation that performance of a plasma focus would progressively improve with progressive reduction of its static inductance $L_{0}$, a recent paper suggests that there is in fact an optimum $L_{\mathrm{o}}$ below which although the peak total current increases progressively the pinch current and consequently the neutron yield of that plasma focus would not increase, but instead decreases. This paper describes the numerical experiments and results that led to this conclusion.
\end{abstract}

\section{Introduction}

A recent paper [1] suggests that for any plasma focus with a fixed capacitance $C_{0}$, there is an optimum static inductance $L_{0}$, below which the focus pinch current $I_{\text {pinch }}$ no longer increases. This paper describes the numerical experiments and results leading to this conclusion of a plasma focus pinch current limitation effect.

We need to say right at the beginning that this $I_{\text {pinch }}$ limitation effect is not the same ${ }^{4}$ as the $I_{\max }$-related mechanism proposed by Nukulin and Polukhin [2] to explain an observed neutron saturation effect. In [2] it is postulated that in large plasma focus devices the peak total discharge current $I_{\text {peak }}$ (which they denote as $I_{\max }$ ) hardly increases with increase in storage energy through increase in bank capacitance $C_{\mathrm{o}}$. This 'tardiness' of $I_{\text {peak }}$ leads to an equation which in the limit of large storage energies $E$ tends towards a constant neutron yield $Y_{\mathrm{n}}$. We state here that [2] deals with a special, though important, class of plasma focus discharge conditions where an increase in $C_{\mathrm{o}}$ needs a corresponding increase in anode length $z_{\mathrm{o}}$. This in turn leads to a situation where the effective discharge impedance, which determines $I_{\text {peak }}$ for any given operating voltage $V_{\mathrm{o}}$, seems to tend towards a constant value as $E$ increases with $C_{\mathrm{o}}$, thus limiting $I_{\text {peak }}$. This work delves deeper into the problem. We show that in another

4 The authors thank a reviewer for stressing that this comparison of our work with that of [2] should be made. 
class of plasma focus discharges, as static inductance $L_{\mathrm{o}}$ is progressively reduced to lower and lower values it is $I_{\text {pinch }}$ (as distinct from and not representative of $I_{\text {peak }}$ ) which becomes limited whilst $I_{\text {peak }}$ continues to increase progressively with no sign at all of reaching a constant value. This remarkable divergence of the values of $I_{\text {pinch }}$ from $I_{\text {peak }}$ indicates then that generally for all classes of plasma focus there is a need to distinguish between the total discharge current $I_{\text {total }}$, which has a peak value $I_{\text {peak }}$, and the actual current driving the plasma dynamics which we call the plasma sheath current $I_{\mathrm{p}}$, denoting its value at the start of the focus pinch phase as $I_{\text {pinch. }}$.

\section{Distinguishing the $I_{\text {total }}$ waveform from the $I_{\mathrm{p}}$ waveform}

A measured trace of $I_{\text {total }}$ is commonly obtained with a Rogowski coil wrapped around the plasma focus flange through which is fed $I_{\text {total }}$ discharged from the capacitor bank between the coaxial electrodes across the back wall. A part of $I_{\text {total }}$, being the plasma sheath current $I_{\mathrm{p}}$, lifts off the back-wall insulator and drives a shock wave axially down the coaxial space. We denote $f_{\mathrm{c}}$ as the current fraction $I_{\mathrm{p}} / I_{\text {total }}$ for the axial phase and $f_{\mathrm{cr}}$ for the radial phases. In modeling it is found that a reasonable value for initial trial for $f_{\mathrm{c}}$ is 0.7 with a similar first trial value for $f_{\text {cr }}$. However in a DPF78 experiment $[3,4] f_{\mathrm{c}}$ was found to vary from 0 at the start of the axial phase rising rapidly above 0.6 for the rest of the axial phase. In the radial phase $f_{\text {cr }}$ was found to stay above 0.6 before dropping to 0.48 at the start of the pinch and then towards 0.4 as the pinch phase progressed. These Stuttgart results confirm a complex relationship between the waveforms of $I_{\text {total }}$ and $I_{\mathrm{p}}$.

The performance of a plasma focus is closely linked to the current $I_{\text {pinch }}$ actually participating in the focus pinch phase rather than the total current flowing in the circuit. It is a common practice to take $I_{\text {peak }}$ or some representative fraction of it as $I_{\text {pinch. Another practice }}$ is to take the value of $I_{\text {total }}$ at the time of the pinch as $I_{\text {pinch }}[2,5]$. Whilst in their special cases this practice could be justifiable, the distinction of $I_{\mathrm{p}}$ from $I_{\text {total }}$ should generally be clearly made. We emphasize that it should be the value of $I_{\mathrm{p}}$ at the time of pinch which is the relevant value for the purpose of yield scaling. The practice of associating yield scaling with the total current waveform (i.e. taking $I_{\text {peak }}$ or $I_{\text {total }}$ at estimated pinch time) would be justifiable if there were a linear relationship between the waveforms of $I_{\text {total }}$ and $I_{\mathrm{p}}$. However as shown by the Stuttgart experiments $[3,4]$ the actual relationship is a very complex one which we may ascribe to the interplay of the various electro-dynamical processes including the relative values of static inductance $L_{\mathrm{o}}$, tube inductance and the dynamic resistances which depend on the tube geometry and plasma sheath speeds. This relationship may change from one machine to the next. Whilst these electro-dynamical processes and other relevant ones such as radiation are amenable to modeling there are other machine effects such as back wall restriking (for example due to high induced voltages during the pinch phase) which can almost unpredictably affect the relationship between $I_{\text {total }}$ and $I_{\mathrm{p}}$ during the crucial radial phases. Hence it is not only simplistic to discuss scaling in terms of the $I_{\text {total }}$ waveform (i.e. taking $I_{\text {peak }}$ or the value of $I_{\text {total }}$ at the estimated time of pinch) but also inconsistent.

One of the most important features of a plasma focus is its neutron production recently reviewed by Vikhrev and Korolev [6]. The well-known neutron yield scaling, with respect to current, based on various compilations of experimental data, is $Y_{\mathrm{n}} \sim I_{\text {pinch }}^{x}$ where $x$ is varied [5, 7-9] in the range 3-5. In a recent paper [10], numerical experiments using a code was used to derive a scaling with $x=4.7$. Difficulties in the interpretation of experimental data ranging across big and small plasma focus devices include the assignment of the representative neutron yield $Y_{\mathrm{n}}$ for any specific machine and the assignment of the value of $I_{\text {pinch. }}$. In a few larger machines attempts were made to measure $I_{\text {pinch }}$ using magnetic probes placed 


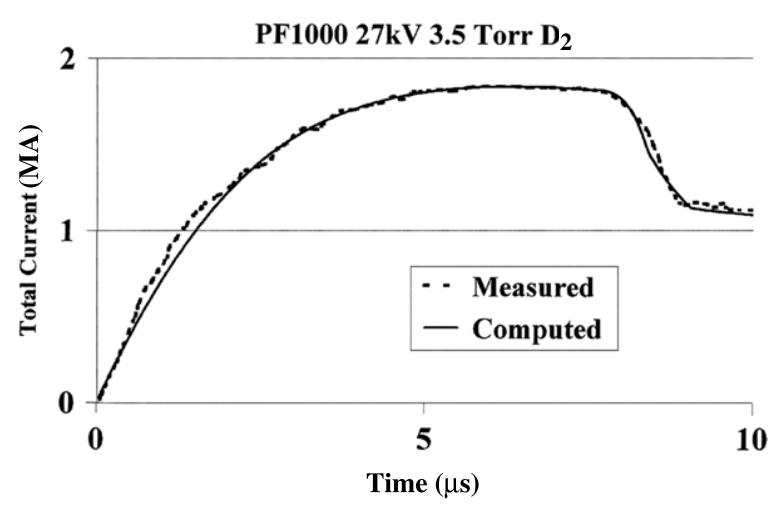

Figure 1. Computed (solid line) versus measured (dotted line) current traces for PF1000 at $27 \mathrm{kV}$, 3.5 Torr $\mathrm{D}_{2}$.

near the pinch region $[3,4,9]$, with uncertainties of $20 \%$. Moreover the probes would have affected the pinching processes. In most other cases related to yield scaling data compilation or interpretation $I_{\text {pinch }}$ is simply assigned a value based on the measurement of peak total current $I_{\text {peak }}$ or the value of total current at the observed current dip.

The difficulties in distinguishing $I_{\text {pinch }}$ from $I_{\text {total }}$ are obviated in numerical experiments using the Lee Model [11-15]. In a typical simulation, the $I_{\text {total }}$ trace is computed and fitted to a measured $I_{\text {total }}$ trace from the particular focus. Three model parameters for fitting are used: axial mass swept-up factor $f_{\mathrm{m}}$, current factor $f_{\mathrm{c}}$ and radial mass factor $f_{\mathrm{mr}}$. A fourth model parameter, radial current factor, $f_{\text {cr }}$ may also be used. When correctly fitted the computed $I_{\text {total }}$ trace agrees with the measured $I_{\text {total }}$ trace in peak amplitude, rising slope profile and topping profile (see figure 1) which characterize the axial phase electro-dynamics. The radial phase characteristics are reflected in the roll-over of the current trace from the flattened top region, and the subsequent current drop or dip. Any machine effects, such as restrikes, current sheath leakage and consequential incomplete mass swept up, not included in the simulation physics is taken care of by the final choice of the model parameters, which are fine-tuned in the feature-by-feature comparison of the computed $I_{\text {total }}$ trace with the measured $I_{\text {total }}$ trace. Then there is confidence that the computed gross dynamics, temperature, density, radiation, plasma sheath currents, pinch current and neutron yield may also be realistically compared with experimental values.

One simplifying feature of the method is that the ratio $I_{\mathrm{p}} / I_{\text {total }}$ is fitted as an average value $f_{\mathrm{c}}$ over the axial phase and separately as another average value $f_{\mathrm{cr}}$ over the radial phase. Whilst an improvement would be to fit some time function of $f_{\mathrm{c}}$ and $f_{\mathrm{cr}}$, our experience is that the present method is adequate to give good agreement for the axial and radial phases up to the end of the plasma focus pinch phase. This ability to fit well has been demonstrated for all classes of machines from the sub-kJ PF400 to high repetition kJ plasma focus NX2 to the medium energy DPF78 all the way through Poseidon and up to the MJ PF1000. The excellent agreement between computed and measured current waveforms for all these machines are available for download from [15].

In a recent paper [5] there was expectation that the large MJ plasma focus PF1000 in Warsaw could increase its discharge current, and its pinch current, and consequently neutron yield by a reduction of its external inductance $L_{\mathrm{o}}$. To investigate this point experiments were carried out using the Lee Model [15]. Unexpectedly, the results indicated that whilst $I_{\text {peak }}$ indeed progressively increased with reduction in $L_{\mathrm{o}}$, no improvement may be achieved due 
to a pinch current limitation effect [1]. This paper reports on the detailed results of these numerical experiments.

\section{The model code used for the experiments}

The Lee Model couples the electrical circuit with plasma focus dynamics, thermodynamics and radiation enabling realistic simulation of all gross focus properties.

The basic model, described in 1984 [11], was successfully used to assist several experiments [16-18]. An improved 5-phase model and code incorporating small disturbance speed [19], and radiation coupling with dynamics assisted other research projects [20-22], and was web-published in 2000 [13] and 2005 [14]. Plasma self-absorption was included in 2007 [13]. It has been used extensively in several machines including UNU/ICTP PFF [16-18, 20, 23], NX2 [21, 22], NX1 [21], and adapted for the Filippov-type plasma focus DENA [24]. A recent development is the inclusion of neutron yield, $Y_{\mathrm{n}}$, using a beam-target mechanism [5], incorporated in the present version [15] of the code RADPFV5.13, resulting in realistic $Y_{\mathrm{n}}$ scaling with $I_{\text {pinch }}$ [10]. The description, theory, code and a broad range of results of this 'Universal Plasma Focus Laboratory Facility' is available for download from [15].

\section{The numerical experiments and discussions}

A measured current trace of the PF1000 with $C_{\mathrm{o}}=1332 \mu \mathrm{F}$, operated at $27 \mathrm{kV}, 3.5$ Torr deuterium, has been published [5], with cathode/anode radii $b=16 \mathrm{~cm}, a=11.55 \mathrm{~cm}$ and anode length $z_{0}=60 \mathrm{~cm}$. In the numerical experiments we fitted external (or static) inductance $L_{\mathrm{o}}=33 \mathrm{nH}$ and stray resistance $r_{\mathrm{o}}=6.3 \mathrm{~m} \Omega$ with model parameters: $f_{\mathrm{m}}=0.14$, $f_{\mathrm{c}}=0.7=f_{\mathrm{cr}}$ and $f_{\mathrm{mr}}=0.35$. The computed current trace (see figure 1 solid line) agrees very well with the measured trace (figure 1 dotted line) through all the phases, axial and radial, right down to the bottom of the current dip indicating the end of the pinch phase. This agreement confirms the model parameters for the PF1000.

Once the model parameters have been fitted to a machine for a given gas, these model parameters may be used with some degree of confidence when operating parameters such as the voltage are varied. We kept these model parameters constant and ran numerical experiments for PF1000 with $C_{\mathrm{o}}=1332 \mu \mathrm{F}$, operating at $35 \mathrm{kV}$ and 3.5 Torr deuterium. We varied $L_{\mathrm{o}}$, from $100 \mathrm{nH}$ in steps of $5 \mathrm{nH}$.

At each $L_{\mathrm{o}}$, ' $a$ ' was adjusted, whilst keeping $c=b / a=1.385$, so that the peak axial speed

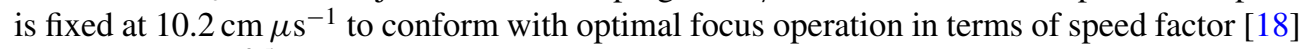
$S=\left(I_{\text {peak }} / a\right) / p^{0.5}$, where $p$ is the operating pressure. Decreasing $L_{\mathrm{o}}$ changes the current rise time as well as effective drive time as shown in figure 2 , which shows three current waveforms corresponding to $L_{\mathrm{o}}=100 \mathrm{nH}$ (current peaking at $1.66 \mathrm{MA}$ ), $L_{\mathrm{o}}=30 \mathrm{nH}$ (current peaking at $2.6 \mathrm{MA}$ ) and $L_{\mathrm{o}}=5 \mathrm{nH}$ (current peaking at $I_{\text {peak }}=4.4 \mathrm{MA}$ ). The experiment for $L_{\mathrm{o}}=5 \mathrm{nH}$ was optimized with $z_{\mathrm{o}}=20 \mathrm{~cm}$ and corresponds to the current trace with current drop starting at around $3 \mu \mathrm{s}$. A fourth trace (for $L_{\mathrm{o}}=5 \mathrm{nH}$ but non-optimal $z_{\mathrm{o}}=40 \mathrm{~cm}$ ) is shown with rise time and peak current almost exactly coincidental with the optimal $L_{\mathrm{o}}=5 \mathrm{nH}$ trace but with a longer drooping top and with current drop starting just after $5 \mu \mathrm{s}$.

As $L_{0}$ was reduced, $I_{\text {peak }}$ increased; and hence ' $a$ ' needed to be increased to maintain optimum $S$. At the same time because of the reducing current drive time, $z_{0}$ needed to be reduced. The geometry inevitably moved from a long thin Mather-type to a shorter fatter geometry (see table 1). Thus whilst $L_{\mathrm{o}}$ and axial section inductance $L_{\mathrm{a}}$ reduced, the pinch inductance $L_{\mathrm{p}}$ increased due to increased pinch length $[1,18]$. 


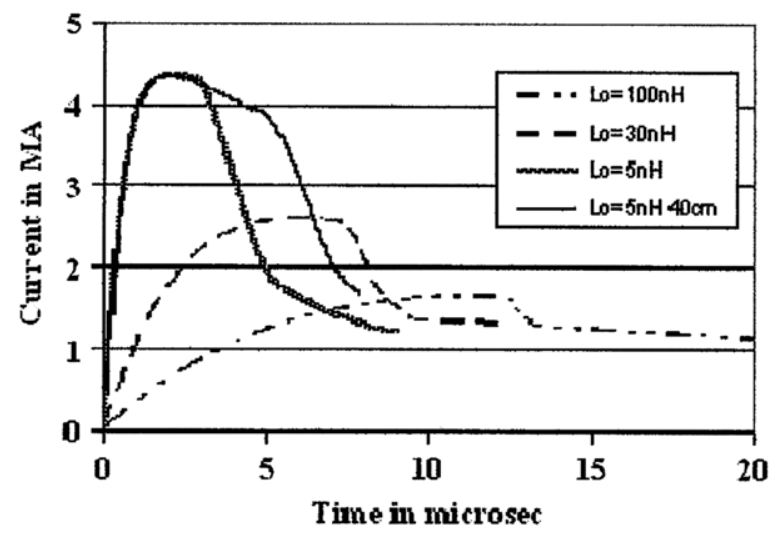

Figure 2. PF1000 current waveforms (computed) at $35 \mathrm{kV}, 3.5$ Torr $\mathrm{D}_{2}$ for a range of $L_{\mathrm{o}}$.

Table 1. Effect on currents and ratio of currents $I_{\text {pinch }} / I_{\text {peak }}$ (computed) as $L_{\mathrm{o}}$ is reduced-PF1000 at $35 \mathrm{kV}, 3.5$ Torr $\mathrm{D}_{2}$.

\begin{tabular}{rlllllll}
\hline $\begin{array}{l}L_{\mathrm{o}} \\
(\mathrm{nH})\end{array}$ & $b$ & $\begin{array}{l}a \\
(\mathrm{~cm})\end{array}$ & $\begin{array}{l}z_{\mathrm{o}} \\
(\mathrm{cm})\end{array}$ & $\begin{array}{l}I_{\text {peak }} \\
(\mathrm{MA})\end{array}$ & $\begin{array}{l}I_{\text {pinch }} \\
(\mathrm{MA})\end{array}$ & $\begin{array}{l}Y_{\mathrm{n}} \\
10^{11}\end{array}$ & $I_{\text {pinch }} / I_{\text {peak }}$ \\
\hline 100 & 15.0 & 10.8 & 80 & 1.66 & 0.96 & 2.44 & 0.58 \\
80 & 16.0 & 11.6 & 80 & 1.81 & 1.00 & 2.71 & 0.55 \\
60 & 18.0 & 13.0 & 70 & 2.02 & 1.03 & 3.01 & 0.51 \\
40 & 21.5 & 15.5 & 55 & 2.36 & 1.05 & 3.20 & 0.44 \\
35 & 22.5 & 16.3 & 53 & 2.47 & 1.05 & 3.20 & 0.43 \\
30 & 23.8 & 17.2 & 50 & 2.61 & 1.05 & 3.10 & 0.40 \\
20 & 28.0 & 21.1 & 32 & 3.13 & 1.03 & 3.00 & 0.33 \\
10 & 33.0 & 23.8 & 28 & 3.65 & 1.00 & 2.45 & 0.27 \\
5 & 40.0 & 28.8 & 20 & 4.37 & 0.97 & 2.00 & 0.22 \\
\hline
\end{tabular}

With large $L_{\mathrm{o}}=100 \mathrm{nH}$ it is seen (figure 2) that the rising current profile is flattened from what its waveform would be if unloaded; and peaks at around $12 \mu \mathrm{s}$ (before its unloaded rise time, not shown, of $18 \mu \mathrm{s}$ ) as the current sheet goes into the radial phase. The current drop, less than $25 \%$ of peak value, is sharp compared with the current rise profile. At $L_{\mathrm{o}}=30 \mathrm{nH}$ the rising current profile is less flattened, reaching a flat top at around $5 \mu \mathrm{s}$, staying practically flat for some $2 \mu \mathrm{s}$ before the radial phase current drop to $50 \%$ of its peak value in a time which is still short compared with the rise time. With $L_{\mathrm{o}}$ of $5 \mathrm{nH}$, the rise time is now very short, there is hardly any flat top; as soon as the peak is reached, the current waveform droops significantly. There is a small kink on the current waveform of both the $L_{\mathrm{o}}=5 \mathrm{nH}, z_{\mathrm{o}}=20 \mathrm{~cm}$ and the $L_{\mathrm{o}}=5 \mathrm{nH}, z_{\mathrm{o}}=40 \mathrm{~cm}$. This kink corresponds to the start of the radial phase which, because of the large anode radius, starts with a relatively low radial speed, causing a momentary reduction in dynamic loading.

Looking at the three types of traces it is seen that for $L_{\mathrm{o}}=100 \mathrm{nH}$ to $30 \mathrm{nH}$, there is a wide range of $z_{\mathrm{o}}$ that may be chosen so that the radial phase may start at peak or near peak current, although the longer values of $z_{0}$ tend to give better energy transfers into the radial phase. Incidentally, this type of trace is the special class considered by Nukulin and Polukhin [2]. For this type of discharge there is sufficient $L_{\mathrm{o}}$ to limit the value of the discharge current until the inductance of the tube, around $1.1 \mathrm{nH} \mathrm{cm}^{-1}$, gets to sufficient value, to in combination with $L_{\mathrm{o}}$ and the dynamic resistance (about $0.6 \mathrm{Ohm}$ per $\mathrm{cm} / \mu \mathrm{s}$ ), determine the value of $I_{\text {peak }}$. In such 


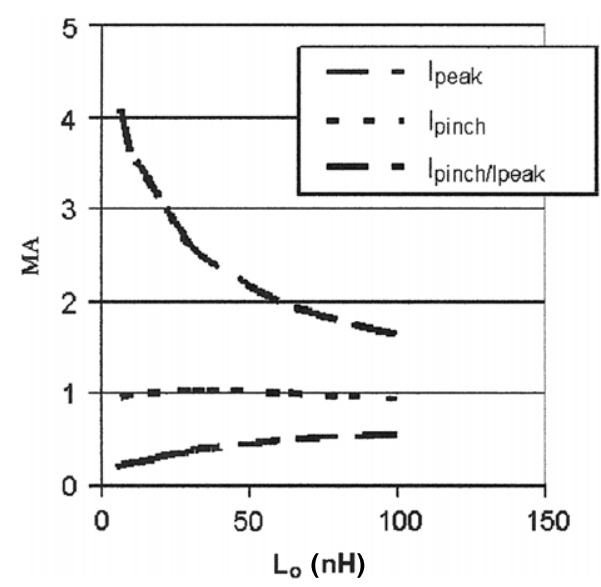

Figure 3. Effect on currents and current ratio (computed) as $L_{\mathrm{o}}$ is reduced-PF1000, $35 \mathrm{kV}$, 3.5 Torr $\mathrm{D}_{2}$.

a case it may be justified in a very rough approximation, by ignoring $L_{\mathrm{o}}$, to say that $I_{\text {peak }}$ is controlled by the full tube inductance, which approximation then results in their conclusion [2] of $I_{\text {peak }}$ (their $I_{\max }$ ) tending towards a constant value with capacitance increase.

At $L_{\mathrm{o}}=5 \mathrm{nH}$, another class of discharge, the situation is quite different. $I_{\text {peak }}$ is much bigger than that calculated using the full tube inductance. This is because with such a small $L_{\mathrm{o}}$, there is a short rise time, 'over-shooting' as it were to a value of current beyond that eventually set when the full tube inductance comes into play (see figure 2). There is hardly any flat top, the current drooping almost immediately from its peak value as the tube inductance increases progressively. For this type of trace, clearly one would not increase length $z_{\mathrm{o}}$ proportionately to increase in $C_{\mathrm{o}}$; since the radial phase should not be delayed too long after early occurrence of $I_{\text {peak }}$. Length matching becomes more critical, although because of the small rate of drop, in terms of optimizing energy transfer to the pinch, a balance has to be sought between increasing storage volume as the axial current sheath advances and the falling current. The computation shows that for $L_{\mathrm{o}}=5 \mathrm{nH}, z_{\mathrm{o}}=20 \mathrm{~cm}$ is optimum, and that $z_{\mathrm{o}}=40 \mathrm{~cm}$ is already significantly off optimum because of the current droop.

At each $L_{0}$, after ' $a$ ' was adjusted for optimum $S$, the computed shape of the current waveform was used as a guide to fine-tune $z_{0}$ for optimum performance, which was finally indicated by the largest $I_{\text {pinch }}$ which corresponds closely to the largest $Y_{\mathrm{n}}$.

The optimized situation for each value of $L_{\mathrm{o}}$ is shown in table 1 . The table shows that as $L_{\mathrm{o}}$ is reduced, $I_{\text {peak }}$ rises with each reduction in $L_{\mathrm{o}}$ with no sign of any limitation. However, $I_{\text {pinch }}$ reaches a broad maximum of 1.05 MA around 40-30 $\mathrm{nH}$. Neutron yield $Y_{\mathrm{n}}$ also shows a similar broad maximum peaking at $3.2 \times 10^{11}$ neutrons. Figure 3 shows a graphical representation of this $I_{\text {pinch }}$ limitation effect. The curve going up to $4 \mathrm{MA}$ at low $L_{\mathrm{o}}$ is the $I_{\text {peak }}$ curve. Although we have numerical data down to $L_{\mathrm{o}}=1 \mathrm{nH}$, in table 1 we only present data from $L_{\mathrm{o}}=5-100 \mathrm{nH}$. At $L_{\mathrm{o}}=1 \mathrm{nH}, I_{\text {peak }}$ reached beyond $6 \mathrm{MA}$ whilst $I_{\text {pinch }}$ dropped further to around $0.95 \mathrm{MA}$.

Thus $I_{\text {peak }}$ shows no sign of limitation as $L_{\mathrm{o}}$ is progressively reduced. However $I_{\text {pinch }}$ reaches a broad maximum. From figure 3 there is a stark and important message. One must distinguish clearly between $I_{\text {pinch }}$ and $I_{\text {peak }}$. In general one cannot take $I_{\text {peak }}$ to be representative of $I_{\text {pinch. }}$.

One may also wish to reflect briefly about the effect on the distribution of energies in the inductances as $L_{\mathrm{o}}$ is lowered. As $L_{\mathrm{o}}$ is lowered from 100 to $1 \mathrm{nH}$, the optimum $L_{\mathrm{a}}$ also 
decreases from around $90 \mathrm{nH}$ to around $6 \mathrm{nH}$. Thus the percentage of energy stored in $L_{\mathrm{a}}$ compared with the total stored inductive energy just before the radial phase has increased from some $47 \%$ to some $86 \%$. It is unfortunate that this increase in available energy is prevented by the interplay of all the physical processes from acting to increase the pinch current. We note that the current fitting process gives us confidence that the computed pinch current is as reliable as if we had measured it; the reliability being the same degree as the reliability of the measurement of the total discharge current, which we used for the current fitting during the computation process. A recent comparison of a numerical experiment with laboratory measurements confirms the reliability of this numerical technique [25].

The above numerical experiments were conducted using a factor RESF $=$ stray resistance/surge impedance of 1.16, fitted for the PF1000. This is a high resistive damping factor for a capacitor bank. We repeated the experiments for PF1000 at $40 \mathrm{kV}$ keeping everything the same except changing RESF to a lower 0.21 . Again $I_{\text {pinch }}$ limitation is clearly seen; at $L_{\mathrm{o}}=60 \mathrm{nH}$ with $I_{\text {pinch }}$ of $1.59 \mathrm{MA}$ and a corresponding $Y_{\mathrm{n}}$ maximum of $8.2 \times 10^{11}$ neutrons.

We carried out several more sets of experiments of which one was with a smaller $C_{\mathrm{o}}=300 \mu \mathrm{F}$, operated at $15 \mathrm{kV}, 6$ Torr D2, with $c=2$ and RESF $=0.1$. We used $f_{\mathrm{m}}=0.08$, $f_{\mathrm{c}}=0.7, f_{\mathrm{mr}}=0.16$ which are the parameters typically fitted for smaller plasma focus such as the NX2 [21,22]. The peak axial speed was kept at $10.3 \mathrm{~cm} \mu \mathrm{s}^{-1}$ for optimal $S$. $L_{\mathrm{o}}$ was reduced from 40 to $1 \mathrm{nH}$. Again a broad maximum was found for $I_{\text {pinch }}$ at $0.455 \mathrm{MA}$ in the range $25-15 \mathrm{nH}$, with a broad maximum for $Y_{\mathrm{n}}$ of $4.4 \times 10^{9}$ neutrons around $L_{\mathrm{o}}=25 \mathrm{nH}$.

Comparing the energy distribution in the circuit elements at the start of the radial phase at the time of $I_{\text {peak }}$ to that near the bottom of the almost linear drop to the time of pinch (see figure 1) the ratio $I_{\text {pinch }} / I_{\text {peak }}$ was derived as a function of $L_{\mathrm{o}}, L_{\mathrm{a}}$ and $L_{\mathrm{p}}$ [1]. This equation and the increasing coupling of the remnant capacitor energy to the pinch were shown to be the physical mechanisms responsible for the decrease in the fraction $I_{\text {pinch }} / I_{\text {peak }}$ and for the pinch current limitation as $L_{\mathrm{o}}$ is progressively reduced, despite the progressive increase in $I_{\text {peak }}$. That theoretical consideration and the results of these numerical experiments together clearly show the importance of distinguishing clearly $I_{\text {pinch }}$ from $I_{\text {peak }}$; and to use $I_{\text {pinch }}$ rather than $I_{\text {peak }}$ as a more consistent quantity for scaling focus yields.

It is also clear that design of new experiments should consider the optimum value of $L_{0}$, rather than the more wasteful practice, technologically speaking, of designing for as low a value of $L_{\mathrm{o}}$ as possible. Moreover, this limitation may now require consideration of new technology to overcome the limitation of $I_{\text {pinch }}$, for example by some form of current stepping [26, 27].

\section{Conclusions}

The results of these sets of numerical experiments indicate that generally corresponding to each plasma focus capacitance of $C_{\mathrm{o}}$, there is an optimum value for $L_{\mathrm{o}}$ below which performance in terms of $I_{\text {pinch }}$ and $Y_{\mathrm{n}}$ does not improve. These experiments confirm the pinch current limitation effect in a plasma focus.

\section{References}

[1] Lee S and Saw S H 2008 Appl. Phys. Lett. 92021503

[2] Nukulin V Ya and Polukhin S N 2007 Plasma Phys. Rep. 33 271-7

[3] Oppenländer T 1981 PhD Dissertation University of Stuttgart, Germany

[4] Decker G, Flemming L, Kaeppeler H J, Oppenlander T, Pross G, Schilling P, Schmidt H, Shakhatre M and Trunk M 1980 Plasma Phys. 22 245-60 
[5] Gribkov V A et al 2007 J. Phys. D: Appl. Phys. 403592

[6] Vikhrev V V and Korolev V D 2007 Plasma Phys. Rep. 33 356-80

[7] Bernard A, Coudeville A, Garconnet J P, Jolas A, Mascureau J de and Nazet C 1978 J. Physique Colloque C1 (suppl 5) $39 \mathrm{C} 1$

[8] Kies W 1988 Laser and Plasma Technology, Proc. 2nd Tropical College (Kuala Lumpur, Malaysia) ed S Lee et al (Singapore: World Scientific) pp 86-137

[9] Herold H 1990 Laser and Plasma Technology, Proc. 3rd Tropical College (Kuala Lumpur, Malaysia) ed C S Wong et al (Singapore: World Scientific) pp 21-45

[10] Lee $\mathrm{S}$ and Saw S H Neutron scaling laws from numerical experiments J. Fusion Energy at press

[11] Lee S 1984 Radiations in Plasmas ed B McNamara (Singapore: World Scientific) pp 978-87

[12] Lee S 1991 IEEE Trans. Plasma Sci. 19912

[13] Lee S 2000/2007 http://ckplee.myplace.nie.edu.sg/plasmaphysics/

[14] Lee S 2005 ICTP Open Access Archive: http://eprints.ictp.it/85/

[15] Lee S Radiative Dense Plasma Focus Computation Package: RADPF http://www.intimal.edu.my/school/fas/ UFLF/

[16] Lee S et al 1988 Am. J. Phys. 5662

[17] Tou T Y, Lee S and Kwek K H 1989 IEEE Trans. Plasma Sci. 17311

[18] Lee S and Serban A 1996 IEEE Trans. Plasma Sci. 24 1101-5

[19] Potter D E 1971 Phys. Fluids 141911

[20] Liu M H, Feng X P, Springham S V and Lee S 1998 IEEE Trans. Plasma Sci. 26 135-40

[21] Lee S, Lee P, Zhang G, Feng X, Gribkov V A, Liu M, Serban A and Wong T 1998 IEEE Trans. Plasma Sci. 261119

[22] Bing S 2000 Plasma dynamics and x-ray emission of the plasma focus PhD Thesis NIE ICTP Open Access Archive: http://eprints.ictp.it/99/

[23] Lee S 1998 Twelve Years of UNU/ICTP PFF—A Review IC, 98 (231) Abdus Salam ICTP, Miramare, Trieste; ICTP OAA: http://eprints.ictp.it/31/

[24] Siahpoush V, Tafreshi M A, Sobhanian S and Khorram S 2005 Plasma Phys. Control. Fusion 471065

[25] Lee S, Saw S H, Lee P C K, Rawat R S and Schmidt H 2008 Appl. Phys. Lett. 92111501

[26] Lee S 1984 J. Phys. D: Appl. Phys. 17733

[27] Saw S H 1991 Experimental studies of a current-stepped pinch PhD Thesis Universiti Malaya 